\title{
Méritos para a sustentabilidade da intervenção em arquitetura urbana na Cova do Vapor, Concelho de Almada (Área Metropolitana de Lisboa, Portugal)
}

\author{
Lina Arroyave \\ l.arroyave@campus.fct.unl.pt \\ Mestranda em Gestão e Políticas Ambientais, Faculdade de Ciência e Tecnologia - \\ Universidade Nova de Lisboa
}

Para citação: ARROYAVE, Lina - Méritos para a sustentabilidade da intervenção em arquitetura urbana na Cova do Vapor, Concelho de Almada (Área Metropolitana de Lisboa, Portugal). Estudo Prévio 16. Lisboa: CEACT/UAL - Centro de Estudos de Arquitetura, Cidade e Território da Universidade Autónoma de Lisboa, 2019. ISSN: 2182-4339 [Disponível em: www.estudoprevio.net]. DOI: https://doi.org/10.26619/2182-4339/16.4

Artigo recebido a 20 de dezembro de 2018 e aceite para publicação a 12 de dezembro de 2019. Creative Commons, licença CC BY-4.0: https://creativecommons.org/licenses/by/4.0/

\section{Resumo}

As diferentes edições do Laboratório de intervenção local em arquitetura in situ/, têm promovido a criação de uma comunidade de prática, onde atores diversificados se encontram e trabalham em torno de um projeto comum. O projeto comum da edição de 2018 foi desenvolvido no bairro de génese informal Cova do Vapor localizado na zona costeira do concelho de Almada, na Área Metropolitana de Lisboa (Município de Setúbal). O referido projeto, visou a proteção da zona dunar, delimitando um acesso à praia para evitar o pisoteio das dunas. O objetivo do artigo é dar destaque, através de indicadores de sustentabilidade, aos méritos de iniciativas como o Laboratório de intervenção local em arquitetura in situ/ Cova do Vapor, relativamente à sua contribuição para as diversas dimensões de sustentabilidade. O indicador utilizado tem o nome de SUSTAIN e, através da sua utilização, chegou-se à conclusão que o melhor resultado que 0 in situ/ Cova do Vapor obteve foi no parâmetro da Sociedade, Tecido Social e Cultura, o que faz com que seja um instrumento mensurável e replicável da gestão adaptativa do território e do ambiente promovendo o bem-estar social.

Palavras-Chave: Comunidade de prática, requalificação urbana, intervenção local, erosão dunar, bairro informal. 


\section{Introdução}

A linha costeira é de grande importância estratégica em termos ambientais, económicos, sociais, culturais e recreativos. No caso português, existe um processo acelerado de ocupação intensiva de muitos lugares costeiros que está em aumento desde a segunda metade do século XX. Segundo Schmidt (2015), no total das freguesias costeiras de Portugal continental a população duplicou desde 1960, e acresceu o número de alojamentos, o que dinamiza e aumenta o processo massivo de 'costeirização'. Este processo é indissociável da evolução dos três principais motores da economia portuguesa das últimas décadas que é o turismo, a construção civil e o imobiliário (Schmidt, 2015) na linha costeira portuguesa.

Neste panorama a gestão e proteção do ambiente e o controlo de riscos costeiros se torna essencial; por isso é fundamental a participação dos stakeholders desde os estágios iniciais e dentro de uma lógica bottom up, dado que pode aumentar a consciencialização e a promoção da integração do conhecimento (Lopes; Videira, 2016). Neste sentido, a abordagem da gestão e proteção do ambiente, e o controlo de riscos costeiros em Portugal, tem de ser feita de diferentes perspetivas e ser deliberativa, principalmente porque Portugal é um dos países europeus mais vulneráveis aos impactos das Alterações Climáticas (Carvalho, 2011), e existe um quadro vulnerável aos riscos ambientais nas suas zonas costeiras (Rocha, 2011).

O Concelho de Almada, possui uma extensa planície costeira arenosa (Pereira Costa, 2015) que integra um vasto sistema de dunas e núcleos urbanos, como a Cova do Vapor, Fonte da Telha e Trafaria (Rocha, 2011). Neste tipo de ecossistemas vulneráveis a riscos, o envolvimento da sociedade é crucial para atingir os objetivos de conservação marinha (Jefferson et al., 2015) porque o seu sistema de dunas tem vindo a mudar desde 1929. A língua de areia, que servia de barreira ao transporte de sedimentos ao longo da costa, recuou cerca de $3 \mathrm{~km}$ e deu-se o rompimento da barreira natural "golada do Tejo" (Rocha, 2011). Após a abertura desta barreira natural, foi urgente executar soluções "pesadas" ou obras de defesa costeira (esporões e muros) na Cova do Vapor (Rocha, 2011); além de soluções "leves" para a defesa costeira, nomeadamente a proteção das dunas através de projetos participativos.

No contexto de soluções "leves", se enquadra o projeto TransforMAR ${ }^{1}$, a Biblioteca do Vapor $^{2}$ e The Dune Project ${ }^{3}$, os quais começaram as suas atividades de proteção dunar de maneira local na Cova do Vapor, um bairro de génese informal no concelho. Estes bairros e territórios urbanos que resultam da ocupação informal, como a Cova do Vapor, têm sido um cenário ideal para iniciativas com lógica bottom up, como o Laboratório de intervenção em arquitetura na comunidade chamado in situ/. O Laboratório in situ/ envolve diversos intervenientes interessados: como habitantes de bairros de génese informal, alunos universitários, arquitetos e entidades públicas (Ramalhete; Silva, 2014).

O Laboratório de intervenção em arquitetura in situ/ nasce com a premissa de resolver problemas reais em comunidades reais, envolvendo atores locais na construção em contexto urbano (Ramalhete; Silva, 2014). A edição de 2018 foi realizada no bairro Cova do Vapor; convidou jovens arquitetos, professores, alunos e 
habitantes do bairro, com o objetivo de delimitar o acesso à praia para evitar 0 pisoteio das dunas e protegê-las.

$\mathrm{Na}$ área de estudo as dunas têm uma função fundamental porque elas servem como proteção em relação ao vento, à rebentação das ondas e ao avanço do mar. Em consequência, proteger as dunas como barreira natural, é uma estratégia da gestão do ambiente costeiro. Mitigar a erosão das dunas delimitando áreas na praia, promove a implementação de soluções práticas para os desafios locais utilizando experiências inovadoras de ensino/aprendizagem.

Parâmetros que medem os méritos das diversas dimensões da sustentabilidade em iniciativas da gestão do Ambiente e território, são fundamentais para replicar experiências de sucesso que integram ambiente, sociedade, educação e território. O termo sustentabilidade está associado ao conceito de desenvolvimento sustentável, no sentido de este último ser o processo que permite atingir ou alcançar a sustentabilidade de um sistema a longo prazo. Assim, a sustentabilidade é um conceito complexo baseado no termo sustentável, que significa "aquilo que pode ser mantido ao longo do tempo" (HEINBERG, 2007) e pode ser medido com diferentes indicadores.

Indicadores de sustentabilidade permitem medir e traduzir quantitativamente os quatro pilares que a definem: governança, ambiente, economia e sociedade; assim, os indicadores de sustentabilidade têm necessariamente de ser de fácil perceção e aplicação em diferentes contextos. Nesse sentido, em 2012 o grupo de trabalho SUSTAIN, desenvolveu um indicador comum como uma ferramenta simples que apoia a autoavaliação e a sustentabilidade costeira em qualquer contexto costeiro europeu; o indicador foi chamado como SUSTAIN. Os indicadores SUSTAIN baseiam-se num método de pontuação que permite ponderar o desempenho de iniciativas em termos de sustentabilidade, principalmente na linha costeira (NUNES et al., 2012) que permite avalizar iniciativas como o Laboratório de intervenção em arquitetura in situ/ Cova do Vapor.

Assim, o objetivo do trabalho é medir quais são os méritos para a sustentabilidade da iniciativa Laboratório de intervenção em arquitetura in situ/ Cova do Vapor, para verificar se é uma experiência que poderia ser replicável e medível em termos de sustentabilidade.

\section{Metodologia}

O Laboratório de intervenção em arquitetura in situ/, na edição 2018, foi desenvolvido na Cova do Vapor, no município costeiro de Setúbal, localizado na linha costeira portuguesa. O Laboratório foi realizado neste local com a participação de atores chave, jovens profissionais com experiência no local, estudantes e professores. $O$ objetivo da iniciativa foi, através do trabalho em equipa e desde a perspetiva bottom up, contribuir com para a diminuição do deterioramento das dunas e evitar o pisoteio na área balnear costeira do bairro de génese informal chamado Cova do Vapor. Ao longo da atuação do Laboratório, foram construídas intervenções na forma de estruturas de madeira de diferentes dimensões localizadas nas coordenadas: 38.662484,-9.258224. As intervenções visavam melhorar o acesso à praia e evitar o 
pisoteio das dunas (Figura 1).

O Laboratório de intervenção em arquitetura in situ/ Cova do Vapor foi realizado nas instalações da Universidade Autónoma de Lisboa e no bairro Cova do Vapor, de 11 a 24 de julho de 2018; foi coordenado pelo Centro de Estudos de Arquitetura, Cidade e Território da Universidade Autónoma de Lisboa (CEACT/UAL) e pelo projeto TransforMAR, com parceria e cofinanciamento da Câmara Municipal de Almada, e o apoio do Leroy Merlin de Almada e da Extruplas.

Para avaliar o desempenho do Laboratório de intervenção em arquitetura in situ/ Cova do Vapor em termos de sustentabilidade, foram aplicadas nove entrevistas (7 mulheres, 2 homens) estruturadas a atores chave envolvidos da iniciativa, participantes ativos da iniciativa, moradores do bairro, estudantes, profissionais e professores; isto com o objetivo de aplicar entrevistas aos participantes da maneira mais abrangente possível. O guião utilizado, que se encontra no ANEXO I, foi aplicado aos entrevistados e esteve baseado no indicador SUSTAIN ${ }^{4}$. Deu-se ainda seguimento aos parâmetros de SUSTAIN os quais estão divididos em quatro componentes de sustentabilidade: (i) Economia, (ii) Ambiente e Recursos Naturais, (iii) Sociedade, Tecido Social e Cultura, e (iv) Responsabilidade Global.

$\mathrm{Na}$ avaliação do desempenho com o indicador SUSTAIN, foram atribuídas pontuações de zero a dez em cada um dos quatro componentes de sustentabilidade, sendo zero o mínimo e dez o máximo (ANEXO I). Cada entrevistado respondeu cada uma das perguntas e pontuou cada um dos quatro componentes de sustentabilidade durante a entrevista. Depois de uma contagem final, foi feita a soma dos valores pontuados por todos os atores chave entrevistados, dando origem a uma agregação da pontuação dividida em 4 valores de síntese por cada componente de sustentabilidade; assim, foram avaliadas todas as entrevistas como uma unidade de resposta. Os méritos do Laboratório foram sintetizados e apresentados num gráfico de 4 eixos, cada eixo representando um componente de sustentabilidade.

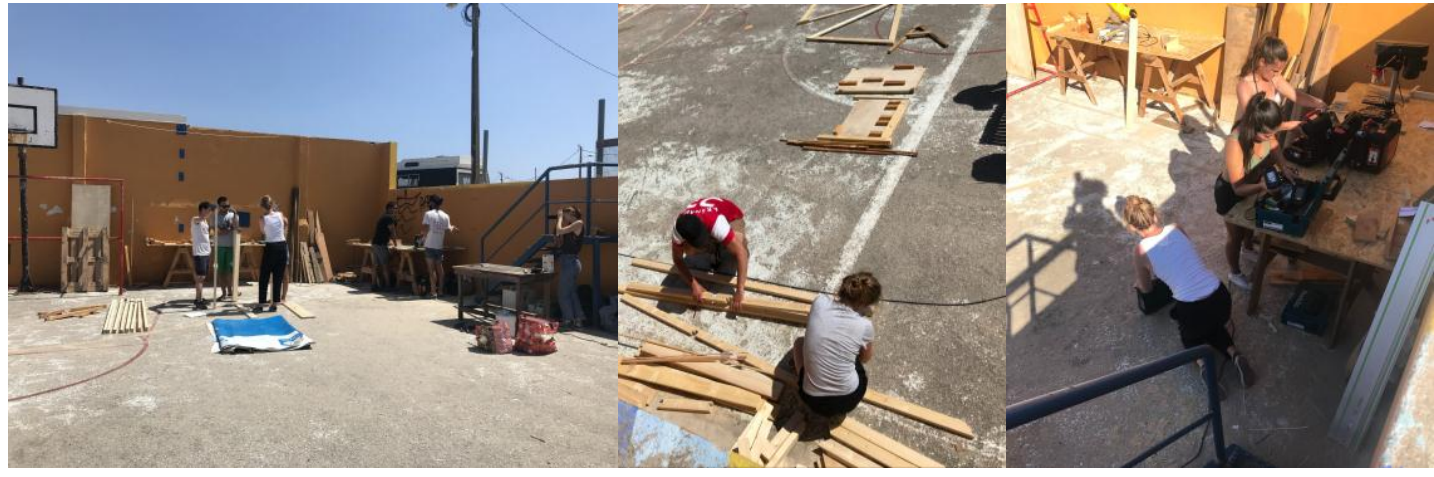




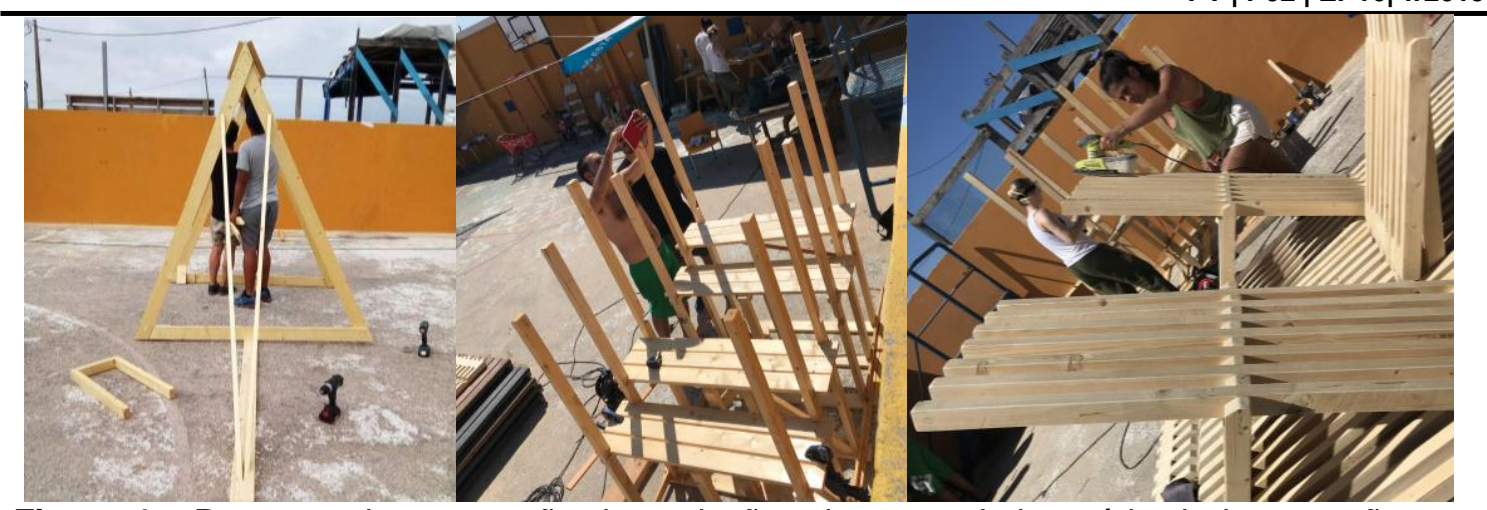

Figura 1 - Processo de construção das soluções durante o Laboratório de intervenção em arquitetura in situ/ Cova do Vapor.

\section{Resultados e discussão}

Através de uma abordagem participativa, foram validados os méritos para a sustentabilidade do Laboratório de intervenção em arquitetura in situ/ Cova do Vapor numa escala local, através do resultado das nove entrevistas aplicadas aos atores chave da iniciativa in situ/ Cova de Vapor. Isto permitiu contribuir para a concretização de novas possibilidades e estratégias de intervenção na comunidade, viradas para o desenvolvimento sustentável e o bem-estar social, através da implementação de soluções e de experiências de ensino/aprendizagem.

Cada um dos quatro méritos tinham um valor total de 400 , assim os resultados das entrevistas com os atores chave da iniciativa, foram tabulados seguindo a metodologia SUSTAIN para obter um valor em cada mérito e calcular qual tinha sido - maior, desde a perspetiva dos atores chave (Tabela 1). O Laboratório de intervenção em arquitetura in situ/ Cova do Vapor obteve o maior mérito para a Sociedade, Tecido Social e Cultura. Isto porque o Laboratório desenvolve a cooperação entre diferentes atores, para além de promover o intercâmbio de conhecimentos e a unidade social ao longo prazo. Na Tabela 1 encontram-se descritos os méritos analisados por cada uma das dimensões da sustentabilidade, onde se explica o impacto de cada mérito na iniciativa in situ/ Cova do Vapor. $\mathrm{Na}$ figura 4, encontra-se o gráfico em que cada eixo corresponde com um dos méritos da sustentabilidade identificados na iniciativa; se observam os diferentes níveis de atribuição identificados em cada mérito da sustentabilidade. 


\section{SOCIEDADE, TECIDO \\ SOCIAL E CULTURA $(203 / 400)$}

O projeto fomenta a requalificação do espaço urbano desde a perspetiva das necessidades locais e reforça o acesso às atividades de recreio porque cria soluções inovadoras que delimitam as diferentes áreas na praia, para além de facilitar o caminho que as pessoas deveriam seguir para não pisotear as dunas. Favorece a aprendizagem ao longo da vida dos atores locais. Promove a cooperação entre o mundo académico, as autoridades locais, os comerciantes e as ONG da área. Também fomenta a integração dos cidadãos e alunos dentro do contexto real. Por outro lado, incentiva a utilização racional e eficiente dos recursos naturais e reciclados, através da utilização direta dos mesmos nas estruturas criadas durante 0 projeto.

\section{ECONOMIA (119/400)}

O projeto melhora as atividades comerciais do local dado que melhora o espaço disponível para o ingresso e uso da praia; espaço que está conectado com locais comerciais. Encoraja ainda a participação dos consumidores, o que melhora as regalias para os bares na praia e vai incentivar, no futuro, a geração de novos empregos.

\section{AMBIENTE E RECURSOS NATURAIS (181/400)}

O projeto contribui para beneficiar os espaços naturais e para proteger o sistema dunar e a sua biodiversidade, o que incrementa a qualidade dos habitats naturais. O projeto encoraja a utilização de produtos reciclados e minimiza a pegada carbónica. Também apoia a articulação do uso das dunas com o sistema de transporte particular porque delimita notavelmente as áreas balneares das áreas de parking, para além de facilitar o acesso à praia e a mobilidade para, por exemplo, pessoas com mobilidade reduzida e crianças em carrinhas de passeio.

\section{RESPONSABILIDADE \\ GLOBAL (181/400)}

O projeto apoia a requalificação do espaço para todas as pessoas que moram e visitam a Cova do Vapor, além de aumentar a consciencialização para uma justiça global. Minimiza 0 impacto das alterações climáticas no sentido de aumentar a consciencialização das pessoas em relação ao risco a través da experiência social e académica. Permite criar uma consciência coletiva entre organizações não governamentais e outras organizações, 0 que pode tornar-se num compromisso conjunto entre municípios e/ou regiões. Também incentiva a comunidade a manterse atualizada e serve de modelo para outros projetos.

Tabela 1 - Méritos de in situ/ Cova de Vapor, relativamente à sua contribuição para as diversas dimensões do desenvolvimento sustentável obtidas com o indicador SUSTAIN 

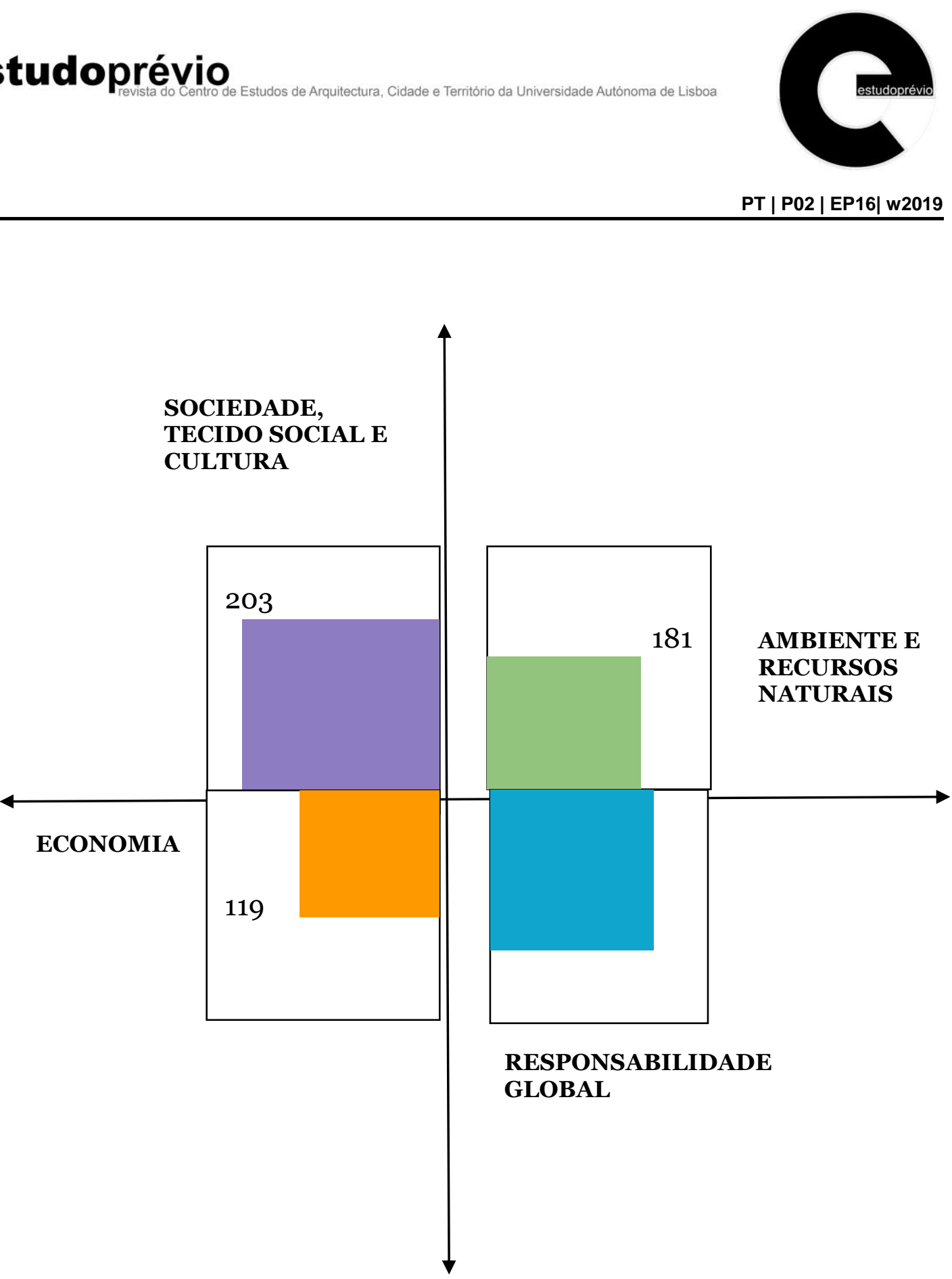

Figura 2 - Representação esquemática de síntese de resultados da análise das quatro componentes do desenvolvimento sustentável no indicador SUSTAIN.

Tendo em conta os resultados obtidos com o indicador SUSTAIN, o Laboratório de intervenção em arquitetura in situ/ Cova do Vapor tem um efeito na sociedade, tecido social e cultura (Figura 2), nomeadamente na justiça espacial, no sentido da distribuição equitativa de recursos, serviços e acesso; considerando-os como um direito para todas as pessoas.

Ressalta-se que o in situ/ Cova do Vapor desenvolveu três intervenções ou construções de madeira (Figura 3) com os parceiros e atores chave envolvidos, para além de atingir os objetivos no que diz respeito à experiência social, académica e arquitetónica com a equipa de participantes. Verificou-se o descrito por Ramalhete et 


\section{estudoprévio}

Estudos de Arquitectura, Cidade e Território da Universidade Autónoma de Lisboa

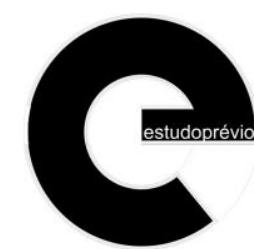

PT | P02 | EP16| w2019

al (2014), em que o aspeto inovador da Intervenção in situ/ Cova do Vapor é que visa projetar e construir soluções para os desafios locais e contribuir para a melhoria na qualidade de vida das populações; para além de apresentar méritos para a sustentabilidade segundo o indicador SUSTAIN.

Ressalta-se que as intervenções estão sempre fortemente inter-relacionadas com a génese dos espaços (Hall, 1998), portanto, o in situ/ é uma oportunidade para propor instrumentos inovadores da gestão adaptativa do território e do ambiente a partir de outras perspetivas que consigam tornar os planos mais estratégicos e eficazes, e menos formais e definidos.

Importa salientar que os parâmetros SUSTAIN utilizados para avaliar os méritos para sustentabilidade de in situ/ Cova do Vapor, constituem também uma forma de comunicação e envolvimento dos atores chave para a identificação de prioridades dentro de um quadro temporal de ações, confirmando assim o afirmado por NUNES et al., (2012).

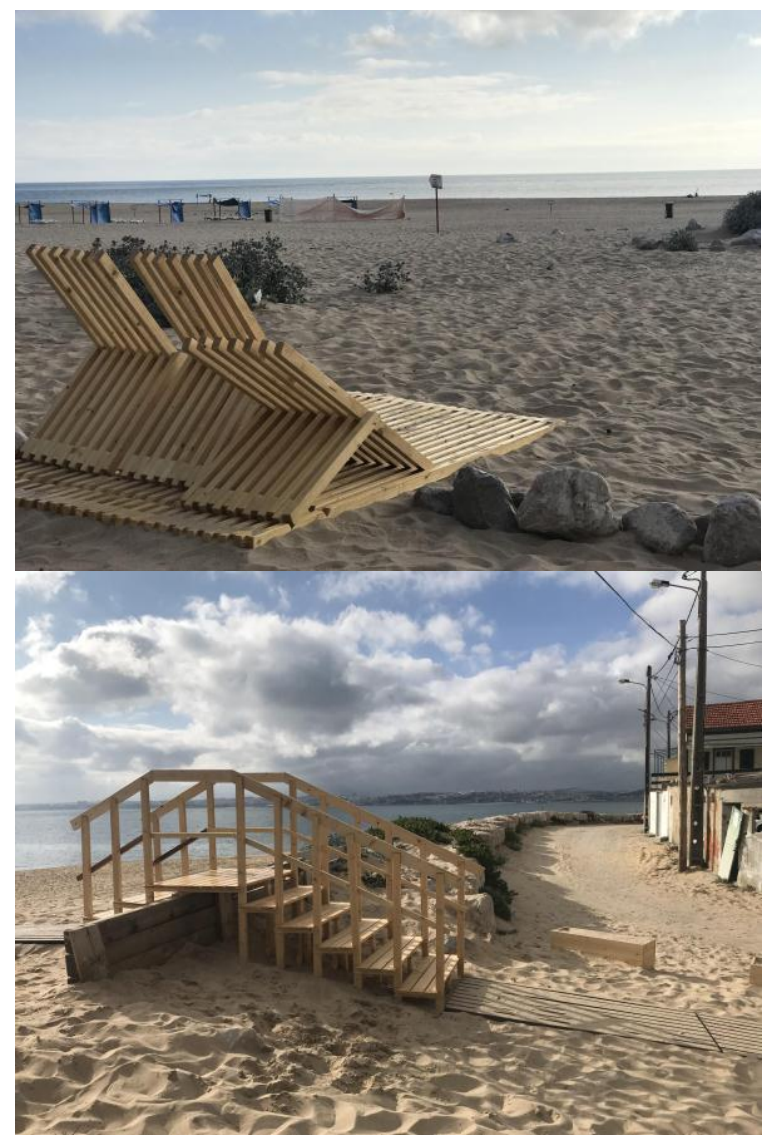




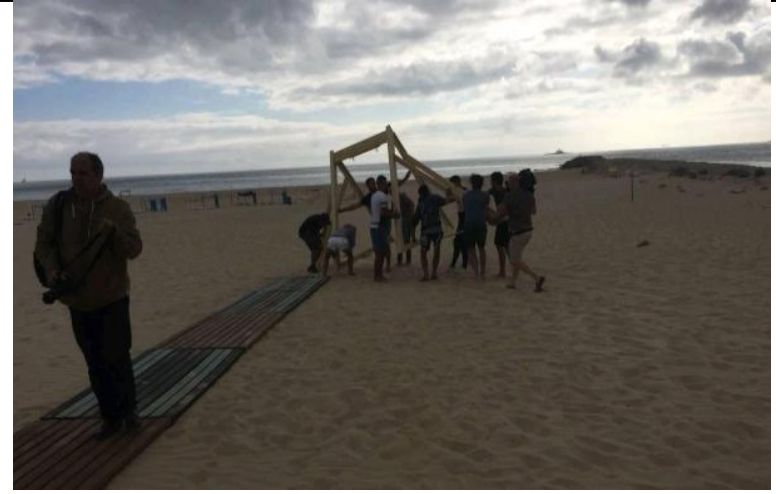

Figura 3 - Intervenções arquitetónicas urbanas realizadas durante Laboratório de intervenção em arquitetura in situ/ Cova do Vapor para a requalificação do espaço e proteção das dunas.

\section{Conclusões}

- A iniciativa in situ/ Cova do Vapor está alinhada com o desenvolvimento sustentável no sentido de "travar a degradação dos solos e travar a perda de biodiversidade", especificamente nas dunas, dado que um dos resultados do projeto foi criar passadiços que facilitarem o acesso às praias e assim evitar o pisoteio no sistema dunar. Entretanto, observou-se que, a maioria (80\%) dos atores chave foram mulheres e raparigas, o que está também alinhado no que diz respeito ao desenvolvimento sustentável, que é: "Alcançar a igualdade de género e capacitar todas as mulheres e raparigas".

- O Laboratório de intervenção em arquitetura in situ/ Cova do Vapor é um exemplo que o ambiente, território e o desenvolvimento são questões inseparáveis e recíprocas. O projeto abre possibilidades de construir em espaços desafiantes com necessidades específicas; e confirma o apresentado por Ramalhete et al (2012) no sentido de pensar em soluções juntamente com diferentes atores interessados e responder às necessidades das pessoas.

- A análise dos méritos para a sustentabilidade do Laboratório de intervenção em arquitetura in situ/ Cova do Vapor demonstrou que este fortalece o movimento de resiliência da comunidade, dado que consegue manter a sua identidade enquanto se adapta aos novos desafios ambientais e sociais.

- Desde a perspetiva buttom up, neste tipo de iniciativas era essencial concretizar um processo mais longo de desenvolvimento do projeto na comunidade local, para assim ter o tempo suficiente de gerar uma participação ativa da população local, quer no âmbito da construção, quer nas atividades relacionadas com a iniciativa in situ/. 
- Identificou-se que é necessário criar mais parcerias para o desenvolvimento deste projeto, por exemplo parcerias com a Agência Portuguesa do Ambiente (APA), com a Comissão de Coordenação e Desenvolvimento Regional de Lisboa e Vale do Tejo (CCDR-LVT), e outras organizações.

- É necessário continuar com um processo paralelo de avaliação no projeto para conhecer e identificar aprofundadamente as estratégias e os benefícios, diretos ou indiretos, de cada edição in situ/ com os seus atores chave.

- O uso do indicador SUSTAIN na iniciativa in situ/ Cova de Vapor, permitiu a aplicação de mecanismos de avaliação; o que poderia ser aplicado a iniciativas similares porque permite validar qualitativamente e qualitativamente o impacto de qualquer iniciativa interdisciplinar, neste caso in situ/.

- Por último, é preciso implementar um dispositivo de autoavaliação SUSTAIN em projetos da gestão do território, desenvolvimento e ambiente, dado que só assim é possível identificar os aspetos a melhorar e abordar as problemáticas com estratégias ajustadas às necessidades específicas, incluindo assim, os componentes da sustentabilidade. A autoavaliação faria com que cada projeto esteja constantemente a rever os seus processos, identificando os seus pontos fortes e aspetos a serem melhorados, para projetar e implementar planos de melhoria.

\section{Recomendações}

- Chama-se a atenção para o facto de in situ/ ser um projeto capaz de gerar inovação socioterritorial numa escala local. No entanto, como desafio para melhorar, era importante integrar mais pessoas da população local, quer na fase de construção, quer na fase de instalação das construções feitas pelos alunos da faculdade de arquitetura, porque pessoas locais poderiam contribuir com conhecimentos que podem favorecer a integração e a aprendizagem contínua e de todas as partes envolvidas. Deste modo, aumentava o número de participantes o que aumentava o número de entrevistas aplicadas para a avaliação. Integrar mais pessoas do local poderia vir a aumentar a integração da comunidade local na construção e instalação, e poderia gerar no seu seio um sentido de pertença com as diferentes peças e um sentimento de relação pessoal, o que facilitaria a manutenção das peças ao longo prazo.

- Tendo em conta o tipo de material com que foram realizadas as peças, madeira, era importante fazer uma visita ao local um tempo após a conclusão da construção para analisar o estado de conservação das peças elaboradas. Assim, ia ser possível aprender sobre o comportamento dos materiais no local e/ou como é possível melhorar. 
- As estratégias de comunicação e publicidade do projeto, poderiam melhorar no sentido de ser reforçadas e as fazer visíveis para o público em geral, para assim elevar o nível de participação e integrar mais participantes possivelmente interessados.

Agradecimentos: Agradecimento pela partilha de informação a Amália Buisson, Eduardo Gomes, Diana Pereira, Rafi Spangenthal, Theo Grainzevelles, Matilda Thomson, Eduardo Conceição, Filipa Ramalhete, José Carlos Ferreira, e estudantes da Universidade Autónoma que participaram no in situ/ Cova do Vapor.

Fotografias: Todas as fotografias do presente texto foram tiradas por Lina Arroyave.

\section{Bibliografia}

CARVALHO A. (org), 2011. As Alterações Climáticas, os Media e os Cidadãos [online]. Coimbra: Grácio Editor. [Consult. 1 Nov. 2019]. Disponível em: http://www.lasics.uminho.pt/ojs/index.php/cecs ebooks/issue/view/143

HEINBERG, R. - Five axioms of sustainability [Em linha]. 2007. [Consult. 04 Jul. 2018]. Disponível em: https://richardheinberg.com/178-five-axioms-of-sustainability

JEFFERSON, R., MCKINLEY, E., CAPSTICK, S., FLETCHER, S., GRIFFIN, H., \& MILANESE, M. (2015). Understanding audiences: Making public perceptions research matter to marine conservation. Ocean and Coastal Management, 115, 61-70. [Consult. 10 Mar. 2019]. Disponível em: https://doi.org/10.1016/j.ocecoaman.2015.06.014

NUNES, M., FERREIRA, J.C, MASCARENHAS, A., RAMOS, T. SUSTAIN, Contribuir para o desenvolvimento sustentável das zonas costeiras. [Em linha]. Lisboa. 2012. [Consult. 04 Mar. 2018]. Disponível em: https://www.apambiente.pt/ zdata/DESTAQUES/2012/ProjetoSUSTAIN/GuiaLicoes Parce riaSUSTAIN/contribuirparadesenvolvimento la.pdf

LOPES, R., \& VIDEIRA, N. (2013). Valuing marine and coastal ecosystem services: An integrated participatory framework. Ocean and Coastal Management, 84, 153-162.

PEREIRA COSTA, M. P. S. Avaliação da vulnerabilidade costeira no concelho de Almada. Dissertação de Doutorado. [Em linha]. Lisboa. 2015. Departamento de Ciências Agrária. Universidade dos Açores. [Consult. 04 Jul. 2018]. Disponível na internet: $<$ https://repositorio.uac.pt/handle/10400.3/3619>.

RAMALHETE, F., PESTANA, J. SILVA, S. Práticas colaborativas e ensino da Arquitetura. José Castro Caldas. [Em linha]. Lisboa. [Consult. 30 Jul. 2018]. Disponível na internet:<

http://www.josecastrocaldas.com/wpcontent/uploads/2012/07/ARTIGO RAMALHETE ET AL final.pdf>.

RAMALHETE, F., SILVA, S. Intervenções Arquitetônicas em espaços informais. Estudo Prévio. [Em linha]. 2014. Artigos. [Consult. 27 Jul. 2018]. Disponível na internet:<

http://www.estudoprevio.net/artigos/43/filipa-ramalhete-sergio-silva.-intervencoesarquitetonicas-em-espacos-informais >. ISSN: 2182-4339.

ROCHA, M. A. Estruturação de áreas com valor ambiental e paisagístico na AML O caso de estudo POLIS Costa da Caparica. Dissertação de Mestrado. [Em linha]. Lisboa. 2011. Universidade de Lisboa. Faculdade de Arquitetura de Lisboa. [Consult. 04 Jul. 2018]. Disponível na internet:<http://hdl.handle.net/10400.5/3466>.

SOJA, E. W. Seeking spatial justice. Minneapolis: University of Minnesota Press, 2010. ISBN 978-0-8166-6668-3. $1^{\text {a }}$ ed. Vol. 16, Pp. 280.

SCHMIDT, L. (2015). Zona Costeira - notas para uma gestão mais integrada - Contextos erosivos, sociais e políticos. In H. G. e J. P. Antunes do Carmo (Ed.), Seminário Inovação 


\section{estudoprévio}

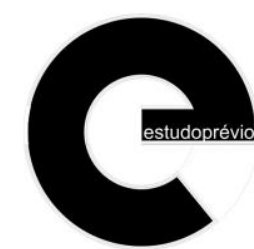

PT | P02 | EP16| w2019

e gestão colaborativa para a sustentabilidade das zonas costeiras. (p. 58). Braga: Universidade do Minho.

\section{Biografia:}

Lina Arroyave é estudante do mestrado em Gestão e Políticas Ambientais na Faculdade de Ciência e Tecnologia, Universidade Nova de Lisboa. Depois de ter trabalhado em conservação da natureza e dos ecossistemas aquáticos, a Lina está focada em encontrar novos instrumentos que facilitem o bem-estar das pessoas e o uso sustentável dos recursos naturais. A Lina tem trabalhado em projetos relacionados com o uso e a conservação da natureza no Instituto Smithsonian em Panamá, no Instituto de Pesquisa em Recursos Biológicos Alexander von Humboldt na Colômbia, em diferentes ONGs na Colômbia, e também foi voluntária no Centro de Pesquisa da Mata Atlântica na Argentina. A Lina também tem experiência na organização de conferências científicas e tesouraria de associações científicas. 
ANEXO I.

Tabela 1. Resultados totais da pontuação dada pelos nove atores chaves durante as entrevistas estruturadas para a obtenção da atribuição dos méritos com 4 componentes da sustentabilidade.

in situ// Cova do Vapor U. Autónoma

24 de Julho de 2018

COMPONENTES DA SUSTENTABILIDADE

Parâmetros de Análise

Pontuação de 0 a 10

1.1 O projeto apoia a economia local e impulsiona os produtos económicos locais e regionais?

1.2 O projeto é gerador de empregos?

1.3 O projeto encoraja a participação dos trabalhadores e melhora as regalias sociais associadas ao emprego?

1.4 O projeto será uma alavanca para aumentar o empreendedorismo e o desenvolvimento de novos produtos e processos produtivos?

1.5 O projeto melhora a centralidade e atratividade de um local?

1.6 O projeto aumenta os benefícios económicos e reduz ao mesmo tempo o consumo de recursos?

1.7 O projeto melhora a situação financeira dos organismos públicos e dos agregados familiares?

1.8 O projeto reduz a necessidade do transporte de pessoas e bens?

$($ Sem Mérito $=0 ;$ Excelente Mérito $=10)$

2.1 O projeto contribui para beneficiar os espaços naturais nas proximidades das zonas urbanas e para apoiar atividades rurais amigas do ambiente?

2.2 O projeto minimiza o consumo de espaço, evita a selagem de solos e o crescimento para o exterior da cidade?

2.3 O projeto previne riscos ambientais e reduz a poluição do ar, da água e dos solos? 
2.4 O projeto reduz os níveis de ruído e a exposição da população ao ruído?

2.5 O projeto diminui o consumo de energia não renovável?

2.6 O projeto reduz o consumo de recursos não renováveis? Encoraja a reutilização de materiais?

2.7 O projeto apoia a biodiversidade e a qualidade dos habitats naturais?

2.8 O projeto apoia a mobilidade sustentável, reduz a mobilidade de desnecessária e articula usos do solo com sistemas de transportes?

SOMA DA PONTUAÇÃO

$($ Sem Mérito = 0; Excelente Mérito = 10)

3 SOCIEDADE, TECIDO SOCIAL E CULTURA

3.1 O projeto contribui para satisfazer necessidades básicas e reduz a pobreza?

3.2 O projeto promove a comunicação e a cooperação entre os cidadãos e destes com as autoridades locais e outras instituições?

3.3 O projeto melhora a qualidade de vida, requalifica o espaço urbano e reforça o acesso aos equipamentos coletivos e aos serviços essenciais?

3.4 O projeto apoia a qualificação dos recursos humanos, a educação e a formação ao longo da vida?

3.5 O projeto promove a participação dos atores locais no seu processo de planeamento e concretização, cria sinergias entre atores e aumenta o capital social?

3.6 O projeto fomenta a integração de cidadãos e de grupos sociais desfavorecidos, é amigo das famílias e da integração de gerações?

3.7 O projeto apoia o bem-estar dos cidadãos, a saúde, o desporto, o consumo responsável e novas atitudes de cidadania?

3.8 O projeto reduz riscos para o homem, contribui para a prevenção da criminalidade e aumenta a perceção de segurança pela população?

$($ Sem Mérito $=0$; Excelente Mérito $=10)$

4 RESPONSABILIDADE GLOBAL

4.1 O projeto fomenta a justiça social e a igualdade de oportunidades entre todos os membros da sociedade?

4.2 O projeto apoia a justiça e a equidade territorial entre as diversas regiões e locais e promove os produtos ecológicos e o comércio justo? 
4.3 O projeto tem em conta os impactos supralocais, gerados sobre outros locais e regiões ainda que distantes?

4.4 O projeto aplica a melhor prática e tecnologia, serve de modelo para outros projetos e promove a troca de conhecimentos?

4.5 O projeto apoia o desenvolvimento de regiões desfavorecidas?

4.6 O projeto aumenta a consciencialização para uma justiça global e sentido de destino conjunto?

4.7 O projeto minimiza as alterações climáticas?

4.8 O projeto apoia a cooperação e intercâmbio entre municípios e regiões?

SOMA DA PONTUAÇÃO

$($ Sem Mérito $=0 ;$ Excelente Mérito $=10)$

${ }^{1}$ O Projeto TransforMar lança a campanha de crowdfunding para apoiar a proteção e restauração ecológica das praias da Cova do Vapor. Ver site http://www.malmada.pt/portal/page/portal/AMBIENTE/AGENDA/DETALHE/?ambiente agenda detalhe $=55$ 7453285\&cboui $=557453285$ e https://www.dunafilms.co.uk/cova

${ }^{2}$ A Biblioteca do Vapor tem um site onde partilha-se informações e eventos da Biblioteca. Ver site https://www.facebook.com/bibliotecadovapor/

${ }^{3}$ Projeto comunitário para a regeneração do cordão dunar da Cova do Vapor. Ver site http://theduneproject.com

${ }^{4}$ Foi adotada a metodologia do SUSTAIN, que permite aos decisores avaliar os méritos das boas práticas, iniciativas e/ou projetos, através da aplicação de uma checklist com os atores principais. Ver no site: Contribuir para o desenvolvimento sustentável das zonas costeiras. Lisboa, novembro.

https://www.apambiente.pt/ zdata/DESTAQUES/2012/ProjetoSUSTAIN/GuiaLicoes Parceria SUSTAIN/contribuirparadesenvolvimento lq.pdf 